International Journal of Applied Mathematics

Volume 32 No. $4 \quad 2019,663-676$

ISSN: 1311-1728 (printed version); ISSN: 1314-8060 (on-line version)

doi: http://dx.doi.org/10.12732/ijam.v32i4.9

\title{
THE SHOOTING METHOD FOR SOLVING SECOND ORDER FUZZY TWO-POINT BOUNDARY VALUE PROBLEMS
}

\author{
Basem S. Attili \\ University of Sharjah \\ College of Sciences - Department of Mathematics \\ Sharjah - P. O. Box 27272 \\ UNITED ARAB EMIRATES
}

\begin{abstract}
We consider the fuzzy two-point boundary value problem (FBVP) subject to some fuzzy boundary conditions on an interval $[a, b]$. Numerically, we start by transforming the two-point boundary value problem into a system of fuzzy initial value problems (FIVP). To solve the resulting system, we use an improved $s$-stage Runge-Kutta Nystrom 4th order method adopted to handle fuzzy problems. Numerical results will be presented to give the numerical details and to show the efficiency of the method.
\end{abstract}

AMS Subject Classification: $65 \mathrm{H} 10$

Key Words: fuzzy two-point BVP's, fuzzy Runge-Kutta method, fuzzy differential equations, shooting method

\section{Introduction}

Many problems in applied sciences and engineering are modeled as fuzzy differential equations (FDE) that are subject to fuzzy initial or boundary conditions, see O'Regan and Lakshmikantham [16]. To obtain a closed form solution for FBVP is generally not an easy task. Instead, numerical approximation is an efficient tool in the simulation of such problems.

In handling FBVP's, different approaches where adopted by different authors. One approach based on Zadeh's extension principle solves the associated crisp problem then substitute the fuzzy conditions in the solution, see Guo et

(C) 2019 Academic Publications 
al. [7] and Zadeh [21]. Another approach transforms the FBVP to a crisp one by interpreting it as a family of differential inclusions, see Hullermeier [8] and Li et al. [15]. A third approach assumes the solution and the derivatives to be fuzzy functions although the boundary values are fuzzy, see Lakshmikantham et al. [14] and O'Regan and Lakshmikantham [16].

Numerically, many authors solved and produced numerical simulations of FBVP's. To list some, the four-stage order Runge-Kutta methods for FDEs were developed in for example Allahviranloo et al. [1], first order FDEs under strongly generalized derivatives were considered by Bede et al. [3]. Generalized Hakuhara differentiability was considered by Chalco-Cano and Roman-Flores [4] to numerically solve a linear second order FBVP. Existence and uniqueness of numerical solutions was considered by Fard et al. [5], while in Fatullayev et al. [6], the authors considered initial value solvers and Gaussian iteration to solve the systems involved. Solving FDE using Bernstein neural network was done by Jafaeri et al. [9] while Adams and Nystrorm methods and predictor-corrector methods for solving FDEs can be found in Khastan and Ivaz [12] and Khastan and Nieto [13] respectively. Euler method was applied for solving initial value problem for FDEs in Palligkinis et al. [17] and Runge-Kutta methods and numerical simulation using general linear method and B-series was done in Rabiei et al. [18] and Rabiei et al. [19], respectively.

We consider the fuzzy two-point boundary value problem of the form, see Gue et al. [7] and Li et al. [15]

$$
y^{\prime \prime}(x)=f\left(x, y(x), y^{\prime}(x)\right), \quad a \leq x \leq b,
$$

subject to the fuzzy boundary conditions

$$
y(a)=\alpha, y(b)=\beta \text {, where } y:[a, b] \times R_{F} \times R_{F} \rightarrow R_{F},
$$

is continuous fuzzy valued function and $\alpha, \beta$ are fuzzy numbers and $R_{F}$ is the set of fuzzy numbers. Numerically, we start by transforming the fuzzy twopoint boundary value problem into a system of fuzzy initial value problems. The missing initial conditions at $x=a$ will then be assumed and we solve the fuzzy initial value problem along the interval. The assumed initial values will then be corrected using the shooting method so that the numerical solution at $x=b$ match the given boundary condition at $x=b$ up to a certain tolerance, for details on the shooting method, see Attili [2], Stoer and Bulirsch [11] and Keller [20]. The outline of this paper will be as follows. In Section 2, we give the basic concepts and definitions of fuzzy numbers and fuzzy differential equations. In Section 3 we explain the shooting method briefly. The extension of the 4th 
order improved Runge-Kutta Nystrom method for treating fuzzy systems is done in Section 4. Finally and in the last section we present some numerical details and results.

\section{Basic concepts}

In order to introduce the fuzzy two point boundary value problem, we need some basic definitions and notations used in fuzzy calculus. In what follows, we will adopt a suitable modification of the Hukuhara differentiability termed as strongly generalized differentiability, which has the advantage of dealing properly with fuzzy differential equations.

Definition 1. (Kaleva [10]) A fuzzy number $u$ is a fuzzy set $u: R \rightarrow$ $[0,1]$ with normal, convex, and upper semi-continuous membership function of bounded support.

We will denote the set of fuzzy numbers on $R$ by $R_{F}$ which means $R \subset R_{F}$. For practical reasons, we use the parametric form of a fuzzy number $u$, denoted by $[u]^{r}$, which is defined as:

$$
[u]^{r}= \begin{cases}\{s \in R: u(s) \geq r\} \quad & \quad r \in(0,1], \\ \overline{\{s \in R: u(s)>0\}} ; \quad r=0,\end{cases}
$$

with $\overline{\{.\}}$ representing the closure of $\{$.$\} . Thus, if u$ is a fuzzy number, then $[u]^{r}=\left[u_{1}(r), u_{2}(r)\right]$, where $u_{1}(r)=\min \left\{s: s \in[u]^{r}\right\}$ and $u_{2}(r)=\max \{s: s \in$ $\left.[u]^{r}\right\}$ for each $r \in[0,1]$. As a result, the following is a characterization of fuzzy numbers that is very convenient in studying fuzzy differential equations. Then $u: R \rightarrow[0,1]$ is a fuzzy number with parameterization given by $\left[u_{1}(r), u_{2}(r)\right]$ defined by $u(s)=\sup \left\{r: u_{1}(r) \leq s \leq u_{2}(r)\right\}$. For the operations on fuzzy numbers, we mainly need to define what is called the Hakuhara difference of two fuzzy numbers $u$ and $v$.

Definition 2. Let $u$ and $v$ are two fuzzy numbers, if there exists an element $w \in R_{F}$ such that $u=v+w$, then $w$ is the Hukuhara difference denoted by $u \ominus v$.

Here $u \ominus v \neq u+(-1) v$. Now based on this definition, we define fuzzy derivatives. 
Definition 3. (Kaleva [10]) Let $y:[a, b] \rightarrow R_{F}$ and $x_{0} \in[a, b]$. We say that $y$ is strongly generalized differentiable at $x_{0}$, if there exists an element $y^{\prime}\left(x_{0}\right) \in R_{F}$ such that either:

(i) for each $h>0$ sufficiently close to 0 , the $H$ - differences $y\left(x_{0}+h\right) \ominus y\left(x_{0}\right)$, $y\left(x_{0}\right) \ominus y\left(x_{0}-h\right)$ exist and

$$
\lim _{h \rightarrow 0^{+}} \frac{y\left(x_{0}+h\right) \ominus y\left(x_{0}\right)}{h}=\lim _{h \rightarrow 0^{+}} \frac{y\left(x_{0}\right) \ominus y\left(x_{0}-h\right)}{h}=y^{\prime}\left(x_{0}\right),
$$

(ii) for each $h>0$ sufficiently close to 0 , the $H$ - differences $y\left(x_{0}\right) \ominus y\left(x_{0}+h\right)$, $y\left(x_{0}-h\right) \ominus y\left(x_{0}\right)$ exist and

$$
\lim _{h \rightarrow 0^{+}} \frac{y\left(x_{0}\right) \ominus y\left(x_{0}+h\right)}{-h}=\lim _{h \rightarrow 0^{+}} \frac{y\left(x_{0}-h\right) \ominus y\left(x_{0}\right)}{-h}=y^{\prime}\left(x_{0}\right) .
$$

Here the limit is taken in the metric space $\left(R_{F}, D\right)$ with $D$ the Hausdorff distance mapping. We say that $y$ is 1 - differentiable on $[a, b]$ if $y$ is differentiable in the sense $(i)$ of the previous definition. Its derivative is denoted by $D_{1}^{1} y$. Similarly, we say that $y$ is $2-$ differentiable on $[a, b]$ if $y$ is differentiable in the sense $(i i)$ of the previous definition. Its derivative is denoted by $D_{2}^{1} y$. Now for the purpose of numerical computations, if we let $y:[a, b] \rightarrow R_{F}$, where $[y(x)]^{r}=\left[y_{1 r}(x), y_{2 r}(x)\right]$ for each $r \in[0,1]$, then $\left[D_{1}^{1} y(x)\right]^{r}=\left[y_{1 r}^{\prime}(x), y_{2 r}^{\prime}(x)\right]$ and $\left[D_{2}^{1} y(x)\right]^{r}=\left[y_{2 r}^{\prime}(x), y_{1 r}^{\prime}(x)\right]$, for more details, see for example Kaleva [10]. This gives two options to find the derivative of a given fuzzy valued function. For the second derivative, we have 4 options given as the derivative of the first derivative; that is, $D_{1}^{1}\left(D_{1}^{1} y(x)\right), D_{2}^{1}\left(D_{1}^{1} y(x)\right), D_{1}^{1}\left(D_{2}^{1} y(x)\right)$ and $D_{2}^{1}\left(D_{2}^{1} y(x)\right)$.

Theorem 4. Let $D_{1}^{1} y:[a, b] \rightarrow R_{F}$ and $D_{2}^{1} y:[a, b] \rightarrow R_{F}$, with $[y(x)]^{r}=$ $\left[y_{1 r}(x), y_{2 r}(x)\right]$ for each $r \in[0,1]$, then:

1. if $D_{1}^{1} y$ is 1 - differentiable, then $y_{1 r}^{\prime}(x)$ and $y_{2 r}^{\prime}(x)$ are differentiable functions and $\left[D_{1,1}^{2} y(x)\right]^{r}=\left[y_{1 r}^{\prime \prime}(x), y_{2 r}^{\prime \prime}(x)\right]$,

2. if $D_{1}^{1} y$ is $2-$ differentiable, then $y_{1 r}^{\prime}(x)$ and $y_{2 r}^{\prime}(x)$ are differentiable functions and $\left[D_{1,2}^{2} y(x)\right]^{r}=\left[y_{2 r}^{\prime \prime}(x), y_{1 r}^{\prime \prime}(x)\right]$,

3. if $D_{2}^{1} y$ is 1 - differentiable, then $y_{1 r}^{\prime}(x)$ and $y_{2 r}^{\prime}(x)$ are differentiable functions and $\left[D_{2,1}^{2} y(x)\right]^{r}=\left[y_{2 r}^{\prime \prime}(x), y_{1 r}^{\prime \prime}(x)\right]$,

4. if $D_{2}^{1} y$ is $2-$ differentiable, then $y_{1 r}^{\prime}(x)$ and $y_{2 r}^{\prime}(x)$ are differentiable functions and $\left[D_{2,2}^{2} y(x)\right]^{r}=\left[y_{1 r}^{\prime \prime}(x), y_{2 r}^{\prime \prime}(x)\right]$. 
This provides a way to transform a fuzzy differential equation into a system of ordinary differential equations and for numerical computation and to use a specific numerical method, there will be no need to use the method in fuzzy setting but rather directly on the resulting ordinary differential system.

\section{The shooting method}

Let be given the two-point boundary value problem

$$
y^{\prime \prime}=f\left(x, y, y^{\prime}\right)
$$

subject to

$$
u(a)=\alpha, \quad u(b))=\beta .
$$

If $f\left(x, y(x), y^{\prime}(x)\right)$ is linear, it can be written in the form

$$
f\left(x, y(x), y^{\prime}(x)\right)=p(x) y^{\prime}(x)+q(x) y(x)+h(x),
$$

in which case the shooting method requires the solution of two linear systems

$$
\begin{aligned}
& u^{\prime \prime}=p(x) u^{\prime}(x)+q(x) u(x)+h(x), u(a)=\alpha, u^{\prime}(b)=0 \\
& v^{\prime \prime}=p(x) v^{\prime}(x)+q(x) v(x), v(a)=0, v^{\prime}(b)=1,
\end{aligned}
$$

and the solution in this case is obtained in one step. Then the solution $y(x)$ is a combination of the two solutions $u(x)$ and $v(x)$ and is given as

$$
y(x)=u(x)+\frac{\beta-u(b)}{v(b)} v(x) .
$$

If $f\left(x, y(x), y^{\prime}(x)\right)$ is nonlinear, we start by transforming (1)-(2) to a first order system of the form

$$
W^{\prime}=\left[\begin{array}{l}
w_{1} \\
w_{2}
\end{array}\right]^{\prime}=\left[\begin{array}{c}
w_{2} \\
f\left(x, w_{1}(x), w_{2}(x)\right)
\end{array}\right]=g(x, W) ; \quad x \in[a, b],
$$

subject to the boundary conditions

$$
w_{1}(a)=\alpha, w_{1}(b)=\beta .
$$

To use the improved Runge-Kutta Nystrom method, let $a=x_{0}<x_{1}<\ldots<$ $x_{m}=b$ be a partition of $[a, b]$. Let $W_{j}\left(x, S_{j}\right)$ denote the solution of the initial value problem

$$
\begin{aligned}
W^{\prime} & =g(x, W) ; \quad W\left(x_{j}\right)=S_{j} ; \\
j & =0,1, \ldots, m-1 .
\end{aligned}
$$


Define the mapping $H: R^{m n} \longrightarrow R^{m n}$ by $H=\left\{H_{j}\right\}$ with

$$
\begin{aligned}
H_{j}= & W_{j}\left(x_{j+1}, S_{j}\right)-S_{j+1} ; \quad 0 \leq j \leq m-2, \\
& \text { and } \\
H_{m-1}= & M_{a} S_{0}+M_{b} W_{m-1}\left(x_{m}, S_{m-1}\right),
\end{aligned}
$$

with $M_{a}=\left[\begin{array}{ll}1 & 0 \\ 0 & 0\end{array}\right]$ and $M_{b}=\left[\begin{array}{ll}0 & 0 \\ 0 & 1\end{array}\right]$. Let $S=\left(S_{0}^{T}, S_{1}^{T}, \ldots, S_{m-1}^{T}\right)$, then (8) can be written in the form

$$
H(S)=0,
$$

which is to be solved for $S_{j}$ using Newton's method given by

$$
S^{(i+1)}=S^{(i)}-\left[D G\left(S^{(i)}\right)\right]^{-1} D G\left(S^{(i)}\right), \quad i=0,1, \ldots,
$$

where $D G\left(S^{(i)}\right)$ is the Jacobian matrix of the system. It should be noted here that there is an equivalence between the shooting (discretized) problem and the original differential equation to be solved in the limit as $m \longrightarrow \infty$. More details on the equivalence and the shooting technique can be found in for example Attili [2], Keller [11] and Stoer and Bulirsch [20] (page 486).

\section{Fuzzy Runge-Kutta Nystrom method of order 4}

The improved $s$-stage Runge-Kutta (IRK) method for solving special second order differential equations was given by Rabiei et al. [18]. The method is a two-step method but requires less number of stages which results in a reduction of function evaluations compared to the normal Runge-Kutta Nystrom method. The general form of the IRK is

$$
\begin{aligned}
& y_{n+1}=y_{n}+\frac{3 h}{2} y_{n}^{\prime}-\frac{h}{2} y_{n-1}^{\prime}+h^{2} \sum_{i=2}^{s} \bar{b}_{i}\left(k_{i}-k_{i-1}\right), \\
& y_{n+1}^{\prime}=y_{n}^{\prime}+h\left[b_{1} k_{1}-b_{-1} k_{-1}+\sum_{i=2}^{s} b_{i}\left(k_{i}-k_{-i}\right)\right],
\end{aligned}
$$

with

$$
\begin{array}{r}
k_{1}=f\left(x_{n}, y_{n}\right), k_{-1}=f\left(x_{n-1}, y_{n-1}\right), \\
k_{i}=f\left(x_{n}+c_{i} h, y_{n}+h c_{i} y_{n}^{\prime}+h^{2} \sum_{j=1}^{i-1} a_{i j} k_{j}\right),
\end{array}
$$




$$
k_{-i}=f\left(x_{n-1}+c_{i} h, y_{n-1}+h c_{i} y_{n-1}^{\prime}+h^{2} \sum_{j=1}^{i-1} a_{i j} k_{-j}\right),
$$

with $i=2, \ldots, s$ and $c_{2}, c_{3}, \ldots, c_{s} \in[0,1], k_{i}$ and $k_{-i}$ depend on $k_{j}$ and $k_{-j}$ for $j=1,2, \ldots, i-1, s$ is the number of function evaluations at each step and depends on the accuracy of the method. Note that $k_{j}$ are evaluated at each step while $k_{-j}$ are calculated from the previous step.

We are interested in the extension of this method to fuzzy differential equations to produce the fuzzy improved Runge-Kutta of order 4 (FIRK). To do so, assume that the approximate solution is given as $[y(x)]^{r}=\left[y_{1}(x ; r), y_{2}(x ; r)\right]$. Define

$$
\left[k_{i}(x ; y(x ; r))\right]^{r}=\left[k_{i 1}(x ; y(x ; r)), k_{i 2}(x ; y(x ; r))\right], i=1, \ldots, s .
$$

As before and at each step,

$$
k_{-i 1}\left(x_{n-1} ; y\left(x_{n-1} ; r\right)\right) \text { and } k_{-i 2}\left(x_{n-1} ; y\left(x_{n-1} ; r\right)\right), i=1, \ldots, s,
$$

are replaced by $k_{i 1}\left(x_{n} ; y\left(x_{n} ; r\right)\right)$ and $k_{i 2}\left(x_{n} ; y\left(x_{n} ; r\right)\right), i=1, \ldots, s$ from previous step. Then the fuzzy improved Runge-Kutta Nystrom method will be given as follows: For $j=1,2$, we have

$$
\begin{aligned}
y_{j}\left(x_{n+1} ; r\right)= & y_{j}\left(x_{n} ; r\right)+\frac{3 h}{2} y_{j}^{\prime}\left(x_{n} ; r\right)-\frac{h}{2} y_{j}^{\prime}\left(x_{n-1} ; r\right) \\
& +h^{2} \bar{b}_{2}\left[k_{2 j}\left(x_{n} ; y\left(x_{n} ; r\right)\right)-k_{-2 j}\left(x_{n-1} ; y\left(x_{n-1} ; r\right)\right)\right] \\
& +h^{2} \bar{b}_{3}\left[k_{3 j}\left(x_{n} ; y\left(x_{n} ; r\right)\right)-k_{-3 j}\left(x_{n-1} ; y\left(x_{n-1} ; r\right)\right)\right] ; \\
y_{j}^{\prime}\left(x_{n+1} ; r\right)= & y_{j}^{\prime}\left(x_{n} ; r\right) \\
& +h\left[b_{1} k_{1 j}\left(x_{n} ; y\left(x_{n} ; r\right)\right)-b_{-1} k_{-1 j}\left(x_{n-1} ; y\left(x_{n-1} ; r\right)\right)\right] \\
& +b_{2}\left[k_{2 j}\left(x_{n} ; y\left(x_{n} ; r\right)\right)-k_{-2 j}\left(x_{n-1} ; y\left(x_{n-1} ; r\right)\right)\right] \\
& +b_{3}\left[k_{3 j}\left(x_{n} ; y\left(x_{n} ; r\right)\right)-k_{-3 j}\left(x_{n-1} ; y\left(x_{n-1} ; r\right)\right)\right],
\end{aligned}
$$

where

$$
\begin{aligned}
k_{11}\left(x_{n} ; y\left(x_{n} ; r\right)\right)= & \min \left[f\left(x_{n}, u\right) ; u \in\left[y_{1}\left(x_{n} ; r\right), y_{2}\left(x_{n} ; r\right)\right]\right], \\
k_{12}\left(x_{n} ; y\left(x_{n} ; r\right)\right)= & \max \left[f\left(x_{n}, u\right) ; u \in\left[y_{1}\left(x_{n} ; r\right), y_{2}\left(x_{n} ; r\right)\right],\right. \\
k_{21}\left(x_{n} ; y\left(x_{n} ; r\right)\right)= & \min \left[f\left(x_{n}+c_{2} h, u\right) ;\right. \\
& \left.u \in\left[v_{11}\left(x_{n} ; y\left(x_{n} ; r\right)\right), v_{12}\left(x_{n} ; y\left(x_{n} ; r\right)\right)\right]\right],
\end{aligned}
$$




$$
\begin{aligned}
k_{22}\left(x_{n} ; y\left(x_{n} ; r\right)\right)= & \max \left[f\left(x_{n}+c_{2} h, u\right) ;\right. \\
& u \in\left[v_{11}\left(x_{n} ; y\left(x_{n} ; r\right)\right), v_{12}\left(x_{n} ; y\left(x_{n} ; r\right)\right)\right],, \\
k_{31}\left(x_{n} ; y\left(x_{n} ; r\right)\right)= & \min \left[f\left(x_{n}+c_{3} h, u\right) ;\right. \\
& \left.u \in\left[v_{21}\left(x_{n} ; y\left(x_{n} ; r\right)\right), v_{22}\left(x_{n} ; y\left(x_{n} ; r\right)\right)\right]\right], \\
k_{32}\left(x_{n} ; y\left(x_{n} ; r\right)\right)= & \max \left[f\left(x_{n}+c_{3} h, u\right) ;\right. \\
& \left.u \in\left[v_{21}\left(x_{n} ; y\left(x_{n} ; r\right)\right), v_{22}\left(x_{n} ; y\left(x_{n} ; r\right)\right)\right]\right],
\end{aligned}
$$

with

$$
\begin{aligned}
v_{11}\left(x_{n} ; y\left(x_{n} ; r\right)\right)= & y_{1}\left(x_{n} ; r\right) \\
& +h c_{2} y_{1}^{\prime}\left(x_{n} ; r\right)+h^{2} a_{21} k_{11}\left(x_{n} ; y\left(x_{n} ; r\right)\right), \\
v_{12}\left(x_{n} ; y\left(x_{n} ; r\right)\right)= & y_{2}\left(x_{n} ; r\right) \\
& +h c_{2} y_{2}^{\prime}\left(x_{n} ; r\right)+h^{2} a_{21} k_{12}\left(x_{n} ; y\left(x_{n} ; r\right)\right), \\
v_{21}\left(x_{n} ; y\left(x_{n} ; r\right)\right)= & y_{1}\left(x_{n} ; r\right)+h c_{3} y_{1}^{\prime}\left(x_{n} ; r\right) \\
& +h^{2}\left\{a_{31} k_{11}\left(x_{n} ; y\left(x_{n} ; r\right)\right)+a_{32} k_{21}\left(x_{n} ; y\left(x_{n} ; r\right)\right)\right\}, \\
v_{22}\left(x_{n} ; y\left(x_{n} ; r\right)\right)= & y_{2}\left(x_{n} ; r\right)+h c_{3} y_{2}^{\prime}\left(x_{n} ; r\right) \\
+ & h^{2}\left\{a_{31} k_{12}\left(x_{n} ; y\left(x_{n} ; r\right)\right)+a_{32} k_{22}\left(x_{n} ; y\left(x_{n} ; r\right)\right)\right\},
\end{aligned}
$$

with the coefficients $c_{2}=\frac{1}{4}, c_{3}=\frac{3}{4}, a_{21}=\frac{1}{32}, a_{31}=0, a_{32}=\frac{9}{32}, b_{-1}=$ $\frac{-1}{18} \cdot b_{1}=\frac{19}{18} \cdot b_{2}=\frac{-1}{6} \cdot b_{3}=\frac{11}{18}$.

\section{Numerical details and examples}

As explained earlier, and to solve the two-point fuzzy boundary value problem, there are 4 different systems to be considered based on the type of second derivatives. They are as follows:

1. The $1-1$ system:

$$
y_{1 r}^{\prime \prime}=f\left(x, y_{1 r}(x), y_{1 r}^{\prime}(x)\right), \quad y_{2 r}^{\prime \prime}=f\left(x, y_{2 r}(x), y_{2 r}^{\prime}(x)\right),
$$

subject to the boundary conditions

$$
y_{1 r}(a)=\alpha_{1 r}, \quad y_{2 r}(a)=\alpha_{2 r}, y_{1 r}(b)=\beta_{1 r}, \quad y_{2 r}(b)=\beta_{2 r} .
$$

Note that the other next three systems are subject to the same boundary conditions given in (13). 
2. The $1-2$ system:

$$
y_{1 r}^{\prime \prime}=f\left(x, y_{2 r}(x), y_{2 r}^{\prime}(x)\right), \quad y_{2 r}^{\prime \prime}=f\left(x, y_{1 r}(x), y_{1 r}^{\prime}(x)\right) .
$$

3. The $2-1$ system:

$$
y_{1 r}^{\prime \prime}=f\left(x, y_{2 r}(x), y_{1 r}^{\prime}(x)\right), \quad y_{2 r}^{\prime \prime}=f\left(x, y_{1 r}(x), y_{2 r}^{\prime}(x)\right) .
$$

4. The $2-2$ system:

$$
y_{1 r}^{\prime \prime}=f\left(x, y_{1 r}(x), y_{2 r}^{\prime}(x)\right), \quad y_{2 r}^{\prime \prime}=f\left(x, y_{2 r}(x), y_{1 r}^{\prime}(x)\right) .
$$

To use the shooting method to solve any of the previous 4 systems, we transform the boundary value problem into an initial value system. This is done by assuming the missing initial conditions; that is, $y_{1 r}^{\prime}(a)$ and $y_{2 r}^{\prime}(a)$, then we solve the resulting system subject to the initial conditions

$$
y_{1 r}(a)=\alpha_{1 r}, \quad y_{2 r}(a)=\alpha_{1 r}, y_{1 r}^{\prime}(a)=S_{1 r}, \quad y_{2 r}^{\prime}(a)=S_{2 r},
$$

using the FIRK method to obtain the solutions $y_{1 r}\left(b ; S_{1 r}, S_{2 r}\right)$ and $y_{2 r}\left(b ; S_{1 r}\right.$, $S_{2 r}$ ). Then using Newtons iteration, we seek the values $S_{1 r}, S_{2 r}$, up to a given tolerance, that satisfies

$$
\begin{aligned}
& y_{1 r}\left(b ; S_{1 r}, S_{2 r}\right)-\beta_{1 r}=0 \\
& y_{2 r}\left(b ; S_{1 r}, S_{2 r}\right)-\beta_{2 r}=0 .
\end{aligned}
$$

Now for numerical testing, we consider the following examples:

Example 1: Consider the fuzzy two point boundary value problem:

$$
\begin{aligned}
y^{\prime \prime}(x)-y^{\prime}(x) & =\rho+1, \quad 0 \leq x \leq 1 \\
y(0) & =0, \quad y(1)=\rho
\end{aligned}
$$

where $\rho$ is a triangular fuzzy number whose membership function is

$$
\rho(s)=\max (0,1-s), s \in R .
$$

The 4 different cases to consider are:

1. The system (12) corresponding to the 1 - 1 differentiability is

$$
\begin{aligned}
& y_{1 r}^{\prime \prime}(x)=y_{1 r}^{\prime}(x)+r, \quad y_{2 r}^{\prime \prime}(x)=y_{2 r}^{\prime}(x)+2-r \\
& y_{1 r}(0)=0, y_{2 r}(0)=0 ; \quad y_{1 r}(1)=r-1, \quad y_{2 r}(1)=1-r .
\end{aligned}
$$



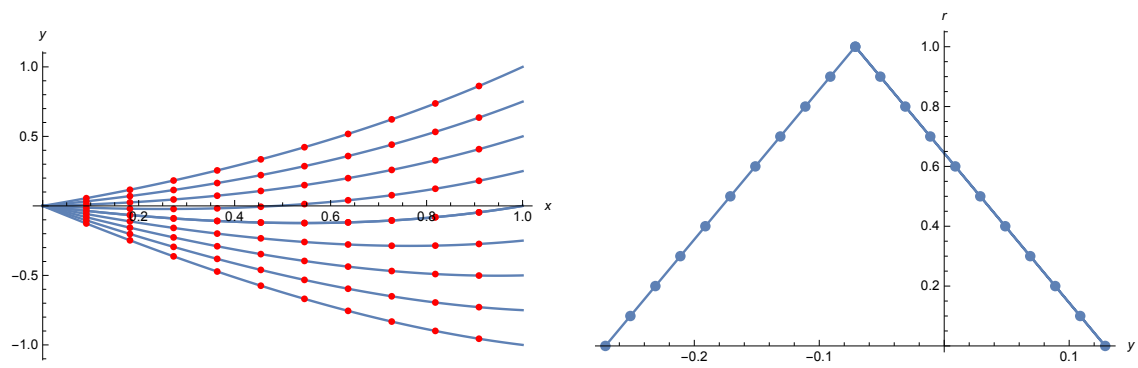

Figure 1: Left: Solutions $y_{1 r}(x)$ and $y_{2 r}(x)$ for different values of $r$; Right:The exact and the numerical solutions at $x=0.2$

2. The system (12) corresponding to the $1-2$ differentiability is

$$
\begin{aligned}
& y_{1 r}^{\prime \prime}(x)=y_{2 r}^{\prime}(x)+2-r, \quad y_{2 r}^{\prime \prime}(x)=y_{1 r}^{\prime}(x)+r \\
& y_{1 r}(0)=0, y_{2 r}(0)=0 ; \quad y_{1 r}(1)=r-1, \quad y_{2 r}(1)=1-r .
\end{aligned}
$$

3. The system (12) corresponding to the $2-1$ differentiability is

$$
\begin{aligned}
& y_{1 r}^{\prime \prime}(x)=y_{1 r}^{\prime}(x)+2-r, \quad y_{2 r}^{\prime \prime}(x)=y_{2 r}^{\prime}(x)+r \\
& y_{1 r}(0)=0, y_{2 r}(0)=0 ; \quad y_{1 r}(1)=r-1, \quad y_{2 r}(1)=1-r .
\end{aligned}
$$

4. The system (12) corresponding to the $2-2$ differentiability is

$$
\begin{aligned}
& y_{1 r}^{\prime \prime}(x)=y_{2 r}^{\prime}(x)+r, \quad y_{2 r}^{\prime \prime}(x)=y_{2 r}^{\prime}(x)+2-r \\
& y_{1 r}(0)=0, y_{2 r}(0)=0 ; \quad y_{1 r}(1)=r-1, \quad y_{2 r}(1)=1-r .
\end{aligned}
$$

To implement the shooting method, we first rewrite each system as a first order initial value system then solve the initial value problems involved using the FIRK. The solutions corresponding to the 1 - 1 differentiability for different values of $r=0.0,0.25,0.5,0.75, \quad 1.0$ are given in Figure 1 (left). Note that the upper 4 curves are for $y_{1 r}(x)$ and the lower 4 curves are for $y_{2 r}(x)$ with the middle for both at $r=1$ since they coincide. Figure 1 (right) shows the solution at $x=0.2$ of both the exact and the approximate numerical one. In Figure 2 we present a $3-\mathrm{D}$ simulation of the solution.

Example 2: Consider the FBVP (see [6]): 


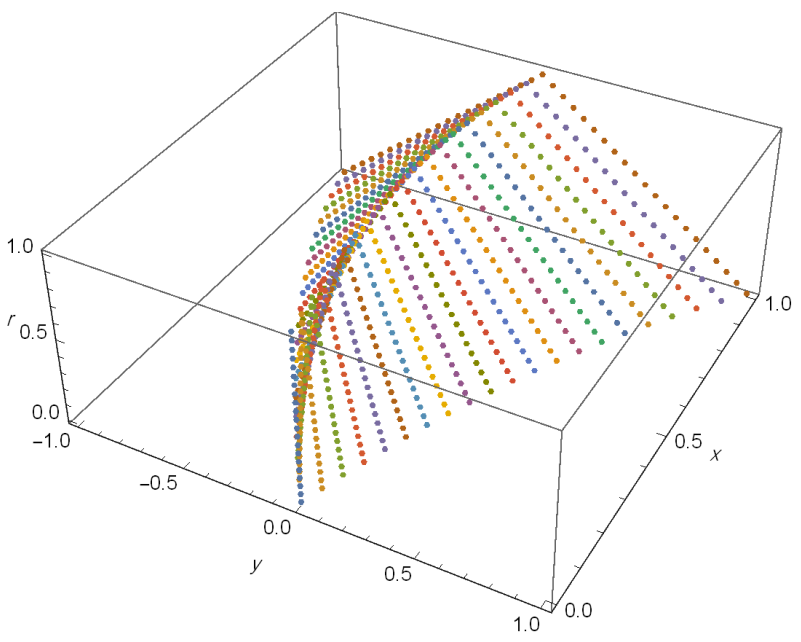

Figure 2: 3-D simulation of the Solution

$$
\begin{aligned}
y^{\prime \prime} & =y^{\prime}+3 y+f(x) \\
y(0) & =\left[\frac{2}{9}(r-1), \frac{2}{9}(1-r)\right] \\
y(1) & =\left[\frac{2}{9}(r-1), \frac{2}{9}(1-r)\right]
\end{aligned}
$$

with

$$
\begin{aligned}
& f_{1 r}(x ; r)=(1-r)(3-2 t)-\frac{1}{3}\left(9 t^{2}-9 t+2\right)(1-r) \\
& f_{2 r}(x ; r)=(r-1)(3-2 t)-\frac{1}{3}\left(9 t^{2}-9 t+2\right)(r-1) .
\end{aligned}
$$

The solutions of the $(1-1),(1-2),(2-1)$ and $(2-2)$ systems for $r=$ 0.5 are given in Figures 3. The results obtained match with that reported in Glufatullayev et al. [6].

\section{References}

[1] T. Allahviranloo, N. Ahmady and E. Ahmady, Numerical solution of fuzzy differential equations by predictor-corrector method, Inform Sci., 177 (2007), 1633-1647. 

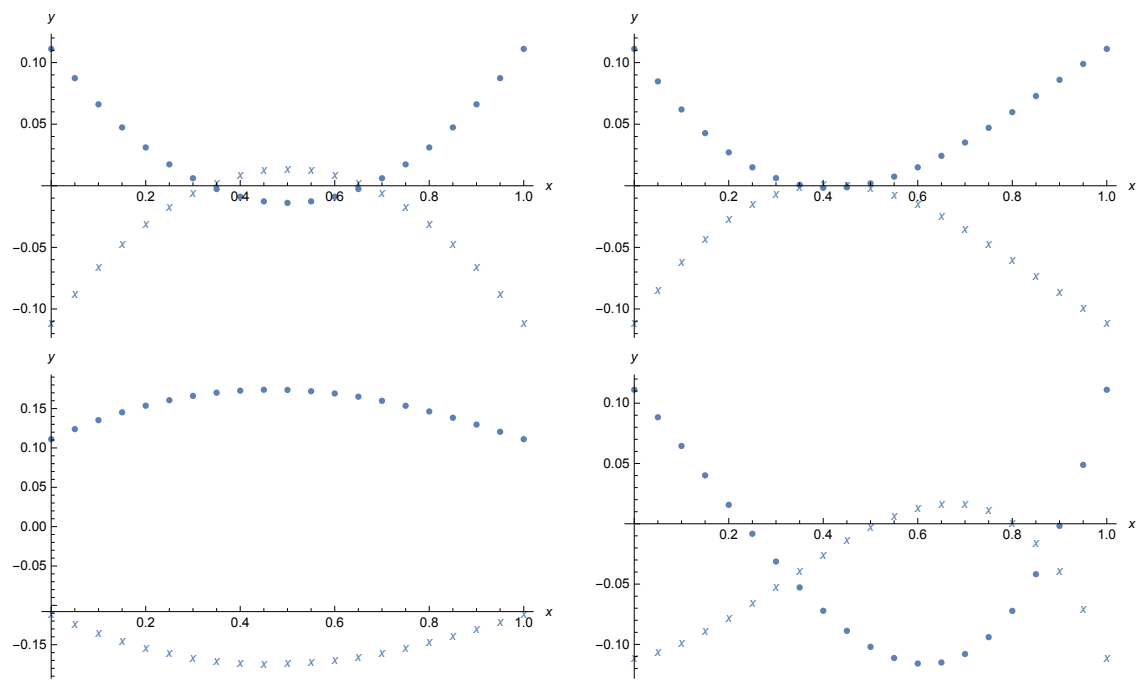

Figure 3: The solutions of the (1-1), (1-2), (2-1) and (2-2) systems for $r=0.5$.

[2] B. Attili, On the numerical implementation of the shooting methods to the one-dimensional singular boundary value problems, Intern. J. Comput. Math., 47 (1993), 65-75.

[3] B. Bede, I. Rudas and A. Bencsik, First order linear fuzzy differential equations under generalized differentiability, Inform. Sci., 177 (2007), 16481662 .

[4] Y. Chalco-Cano, and H. Roman-Flores, On new solution of fuzzy differential equations, Chaos, Solit. Fract., 38 (2008), 112-119.

[5] O. Fard, T. Bidgoli and A. Rivaz, On existence and uniqueness of solutions to the fuzzy dynamic equations on time scales, Math. Comput. Appl., 22 (2017), 1-16.

[6] A. G. Fatullayev, E. Can and C. Koroglu, Numerical solution of a boundary value problem for a second order fuzzy differential equation, TWMS J. Pure Appl. Math., 4 (2013), 169-176.

[7] X. Guo, D. Shang and X. Lu, Fuzzy approximate solutions of second order fuzzy linear boundary value problems, Boundary Value Problems, 2013 (2013), Art. 212; DOI: 10.1186/1687-2770-2013-212. 
[8] E. Hullermeier, An approach to modeling and simulation of uncertain dynamical systems, Inter. J. Uncertain. Fuzz. and Knowledge Based Syst., $\mathbf{5}$ (1997), 117-137.

[9] R. Jafari, W. Yu, Xiaoou Li and S. Razvarz, Numerical solution of fuzzy differential equations with Z-numbers using Bernstein neural networks, Intern. J. Comput. Intelli. Sys., 10 (2017) 1226-1237.

[10] O. Kaleva, Fuzzy differential equations, Fuzzy Sets and Systems, 24 (1987), 301-317.

[11] H.B. Keller, Numerical Solution of Two Point Boundary Value Problems, SIAM (1976).

[12] A. Khastan and K. Ivaz, Numerical solution of fuzzy differential equations by Nystrom method, Chaos, Solit. Fract., 41 (2009), 859-868.

[13] A. Khastan, and J. Nieto, A boundary value problem for second order fuzzy differential equations, Nonlinear Anal., 72 (2010), 3583-3593.

[14] V. Lakshmikantham, K. Murty and J. Turner, Two point boundary value problems associated with nonlinear fuzzy differential equations, Math. Inequal. and Applic., 4 (2001), 527-533.

[15] D. Li, M. Chen and X. Xue, Two point boundary value problems of uncertain dynamical systems, Fuzzy Sets and Systems, 179 (2011), 50-61.

[16] D. O'Regan, V. Lakshmikantham and J. Nieto, Initial and boundary value problems for fuzzy differential equations, Nonlin. Anal., 54 (2003), 405415.

[17] S. Palligkinis, G. Papageorgiou and I. Famelis, Runge-Kutta methods for fuzzy differential equations, Appl. Math.and Comput. 209 (2009), 97-105.

[18] F. Rabiei, F. Ismail, N. Senu and N. Abasi, Construction of improved Runge-Kutta Nystrom method for solving second order ordinary differential equations, World Appl. Sci. J. 20 (2012), 1685-1695.

[19] F. Rabiei, F. Abd Hamid, M. Rashidi and F. Ismail, Numerical simulation of fuzzy differential equations using general linear method and B-series, Advanc. in Mech. Eng., 9 (2017), 1-16.

[20] J. Stoer and R. Bulirsch, Introduction to Numerical Analysis, SpringerVerlag, New York (1980). 
[21] L.A. Zadeh, The concept of a linguistic variable and its applications in approximate reasoning, Inform. Sci., 8 (1975), 199-249. 